\title{
The Impact of MOOC E-learning on Communication Engineering
}

\author{
Fang Dong ${ }^{1, a}$, Junbiao Liu ${ }^{2, b}$, Hong Liu ${ }^{1, c}$, Shenyi Dai ${ }^{3, d}$ \\ ${ }^{1}$ School of Information \& Electrical Engineering, Zhejiang University City College, Hangzhou, \\ 310015, China \\ ${ }^{2}$ Hangzhou Bicone Technology Co., Hangzhou, 310007, China \\ ${ }^{3}$ College of Economics and Management, China Jiliang University, Hangzhou, 310018, China \\ adongf@zucc.edu.cn, bemail:hzclive@163.com, 'liuhong@zucc.edu.cn, ${ }^{d}$ daishenyi@cjlu.edu.cn
}

Keywords: MOOC; Communication Engineering; E-learning; Big Data; Pocket Lab

\begin{abstract}
According to the characteristics of communication engineering, the e-learning method known as MOOC is performed in basic and professional courses to improve education effect. A progress of MOOC practice is presented, and then its specific impact on several curricula in four colleges and universities is introduced. Based on the applications of big data, MOOC platform is able to dynamic assess the learning level of students, facilitating teachers to pace their teaching and adjust the teaching method. Furthermore, with the help of pocket lab and professional software, MOOC can get the best teaching effect that students master the ability of application and innovation in communication engineering.
\end{abstract}

\section{Introduction}

MOOC [1] [2] stands for Massive Open Online Course, which is proposed by Cormier Bryan and Alexan Dave on the basis of network experimental teaching in 2008. Using the network platform and broadband video on demand, MOOC provide publicly high-quality teaching resources to the whole society, especially giving the registered users of MOOC platform a complete learning experience. Since the concept of MOOC was proposed, it has been rapidly popularized all over the world, and numerous platforms are emerging. In 2012, three major universal MOOC platforms, named Coursera, edX and Udacity, respectively, have been put into operation [3]. Soon afterwards Chinese local MOOC platforms (MOOC College, Tsinghua School Online and so forth) have also launched a number of sharing curricula of quality. By the beginning of 2015, there are more than 1100 MOOC platforms are still running [4].

In addition to MOOC, there are other online education platforms, such as Open Course Ware (OCW) proposed by MIT in 2008 [5]. Interesting distinctions can be drawn between the two platforms. MOOC is weighted in course online that the audiences feel the teacher is teaching themselves, meanwhile OCW tends to classroom online that the audiences watch the teacher giving his students lectures via Internet. Compared with TV University [6], MOOC platform has more interactive teaching content with teachers or teaching assistants, including built-in test function, questions \& answers of learning community and online course assignments. Due to the fully openness of MOOC, any general course can find a large amount of curriculum resources on various platforms, and then set up the flipped classroom / inverted classroom [7]. Therefore, Stanford University is aim to become an Open Loop University in 2025 [8].

MOOC is the product of the big challenge when traditional curricula affronts Internet and big data, which redefines the formal college education. Three types of relationship exist between MOOC and face-to-face class, i.e. substitution, competition and cooperation.

In this paper a new optimal design of soccer robot control system which is based on mechanical analyses and calculations on the pressure and transmutation states of chip kick mechanics, this new control system with high precision for speed control and high dynamic quality. Most studies suggest that MOOC practice the knowledge points rather than teaches them, and thus it is very suitable for the teaching of engineering courses [9]. 


\section{Characteristics of Curricula in Communication Engineering}

Communication engineering is one of the key industries in China, which aims to cultivate a number of experts professional in communication equipment's research, development, application and technical service. The major in many colleges usually offers several specialties, such as digital communication and network communication, focusing on practice training and vocational skill acquiring.

The basic and professional courses in the major require students to have a certain mathematic, logical and English ability. Unfortunately, that is not necessarily the case. In fact, the reform or the practice in the course often encounters the following challenges: (1) Fragmentation of knowledge points makes the weak student fail to reach the required standard. In addition, the teaching time of theoretical course is compressed with the same teaching aim, which makes matters worse. (2) The theoretical courses divorce from the corresponding practical / experiment courses, and course selection make both of them not related to each other. (3) Student engagement is low in the learning progress, and the interaction between teachers and students is insufficient. Most of course platform is only used for online quizzes and exercises. (4) Theory derivation is still dominant in the teaching, neglecting a wide range of communication applications, so that students are unable to link the two together。

In order to improve the education effect of communication engineering courses, a thorough teaching support environment is needed, including: (1) complete teaching videos of knowledge points, (2) a set of online tests with various level, (3) a list of the emphases and difficulties of the course, (4) sufficient supplementary materials and application examples, (5) statistics of each student's learning process and the course, (6) BBS community for teachers and students to discuss and Q \& A in real time, (7) resources sharing among teachers and student. All of them can be achieved by MOOC and the flipped classroom. Fragmentation of knowledge points in communication engineering courses is very suitable for recording MOOC teaching videos, online tests, materials and examples can be supported by MOOC platform, and pocket lab (as shown in Fig.1 available at \$15) and professional software allow MOOC to integrate theoretical and practical courses.

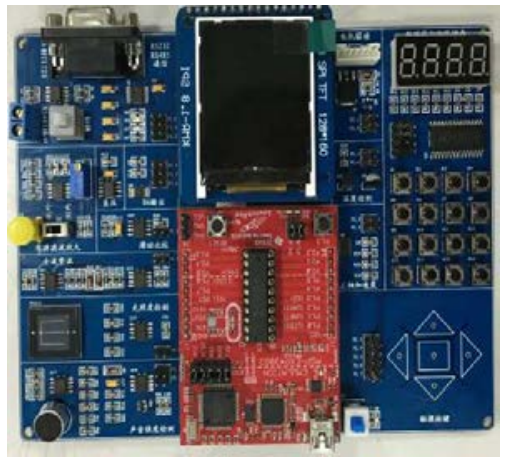

(a) pocket lab of MCU

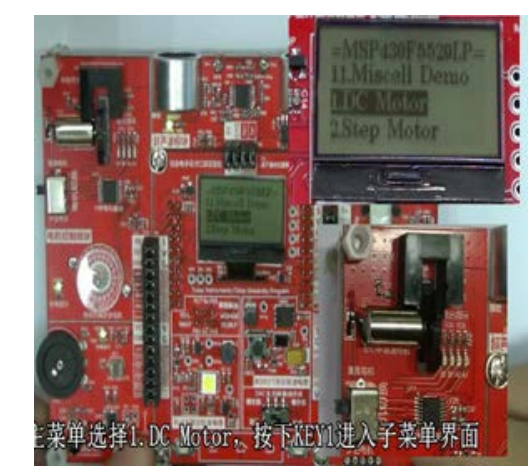

(b) pocket lab of Analog Electronic

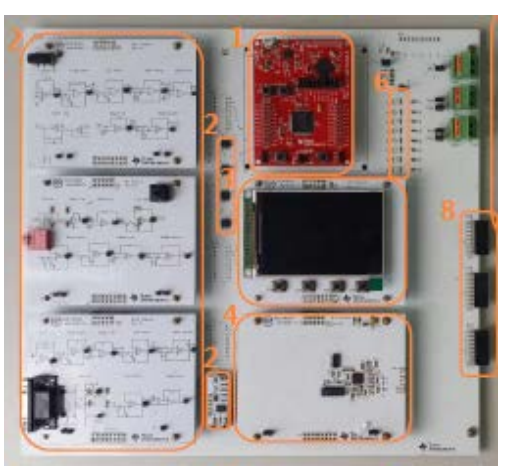

(c) pocket lab of signal

Fig.1. Pocket lab of communication engineering curricula

Combined with online learning and face-to-face learning, MOOC-based learning method can effectively relate theoretical knowledge in the classroom and extra-curricular application, consequently it can obtain the best teaching effect to enhance the students' employment or entrepreneurship.

\section{Progress of MOOC Practice}

The progress of MOOC practice in communication engineering is shown in Fig.2, consisting of the division of knowledge points, video recording and exercise preparation, online learning activities, etc. If there is flipped classroom, student grouping, discussion of knowledge points and experiment design should also be taken into account. 


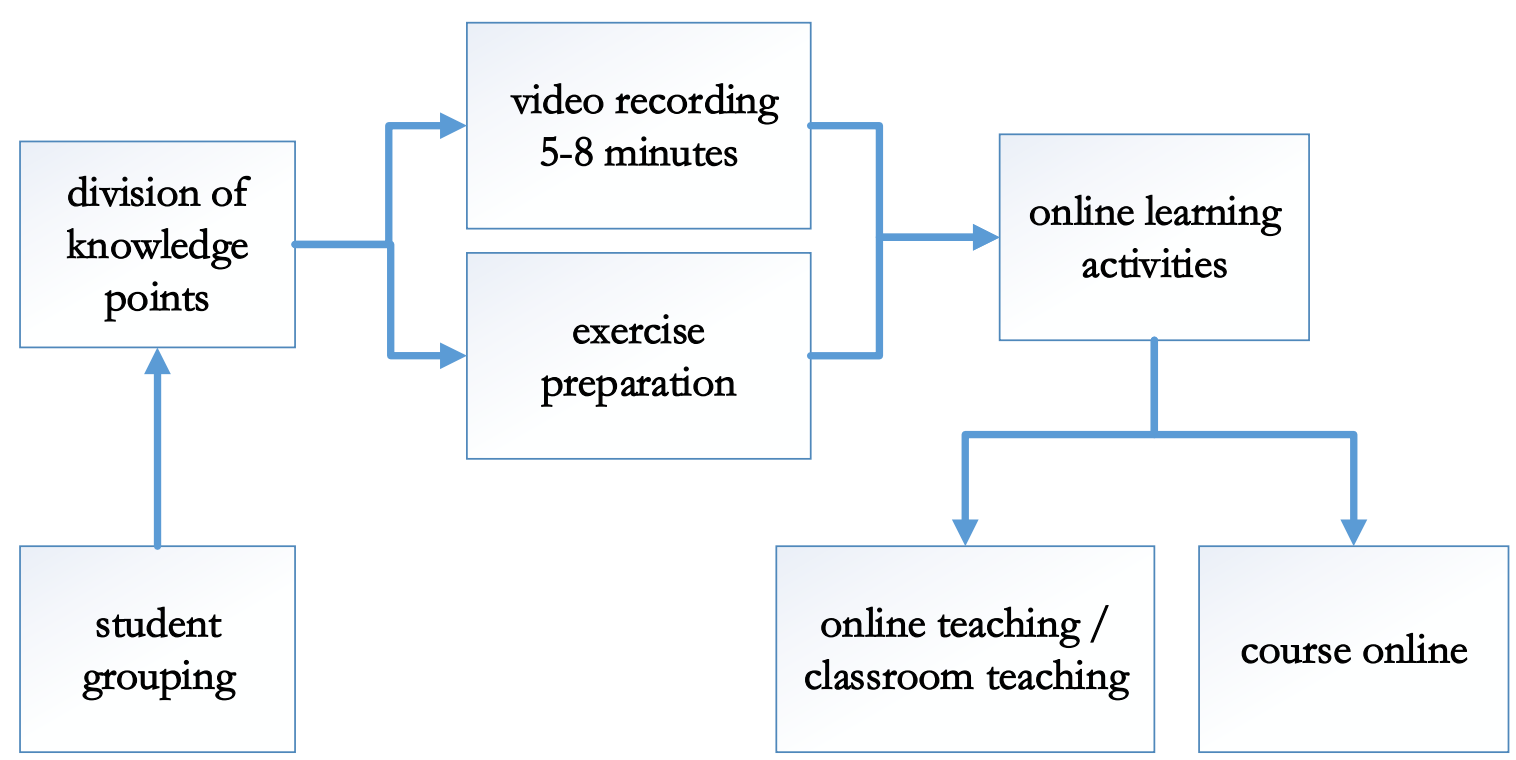

Fig.2. Progress of MOOC practice in communication engineering

In the early stage of MOOC construction, in order to meet the requirements of multi-level teaching, course contents need to be made an overall plan according to the major and the type of students, whose schedule is planned for a week based on units of knowledge points. For example, a knowledge point in Chapter 7 of 'Digital Signal Processing' course is 'the effect of finite word length'. Students from other majors are not required to learn the point, and it does not affect other content learning. For capable students, they can select 'coefficient quantization in digital filters' experiment in the pocket lab by themselves to expand the knowledge point.

In MOOC teaching step, students at first acquire knowledge points via watching videos to complete self-study. In order to ensure the effectiveness of learning, each video should be limited in 5-8 minutes. Moreover, different recording methods is chosen in view of the category of points: (1) concepts using PPT to explain, (2) applications using pocket lab or professional software to simulate. For example, in 'Analog Electronic Design' course, the circuit of ideal amplifier uses PPT to introduce the point, whereas programmable gain amplifier commonly used in practice shows the ability of switch in programming through professional software such as Multisim or EDA.

An important difference between MOOC and traditional teaching is that there is a wealth of online learning activities, increasing exchange with students and teachers. In addition to the general online Q \& A and learning community, MOOC platform can also set special topics of academic discussion. For example, in 'Principles of Circuits' course, teachers can set 'how to measure frequency response of RC circuit' or 'how to get a sine wave generator' experiment on MOOC platform as homework or topic of class discussion, and therefore theoretical and practical courses come together.

\section{Effect of the Application of MOOC in Communication Engineering}

Courses with MOOC in communication engineering have now turned into a full-scale reform throughout all levels of colleges and universities. Basic course 'Principles of Circuits' is used as an example to exhibit the practice methods and their effect of the application of MOOC on several university curricula.

Tsinghua University: The freshmen choose MOOC class in the second semester via course-selected system. Class size is 20 students, including theoretical and experimental courses. The arrangement of a single face-to-face class is : (1) 10-15 MOOC videos of knowledge points are discussed, which involves $40-50 \%$ time; (2) the exercises assigned in advance are revealed answers and a more challenged engineering practice is discussed, which involves $20-40 \%$ time; (3) the corresponding MOOC experiment is illustrated and results are demonstrated, which involves 
$10-40 \%$. After using MOOC, the teaching assessment of the course is ranked in the top 5\% among all courses in the university, as shown in Fig.3

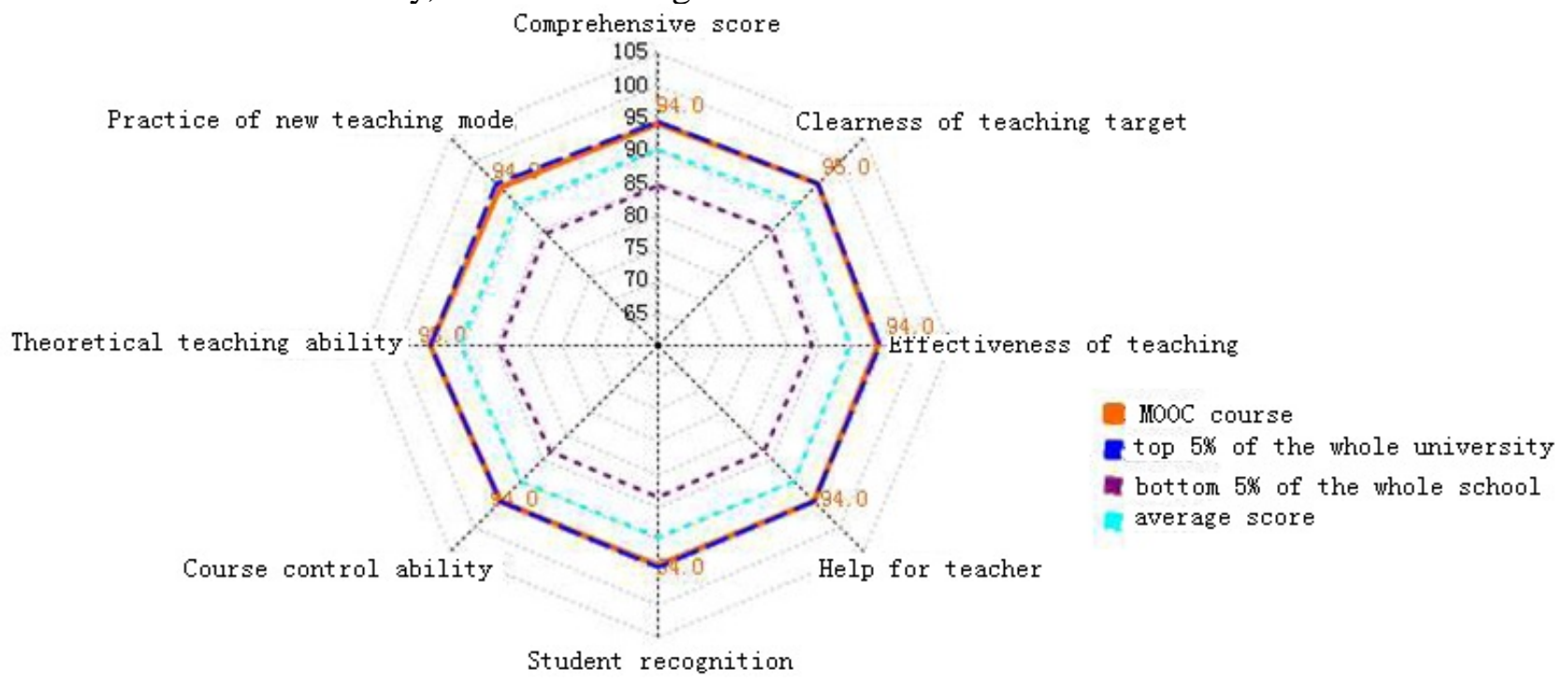

Fig.3. Effect of the application of MOOC in 'Principles of Circuits' in Tsinghua University

Nanjing University: Nanjing University randomly selects freshmen in the first semester to join MOOC class. Class size is 30-40 students, and they are divided into six learning group. The class also combine theoretical course with experimental course. The arrangement of a single face-to-face class is : (1) reflect questions are released on MOOC platform before class, which will be discussed or stated in the class; (2) the design scheme and its circuit of a certain application are reported by a learning group; (3) an experiment campaign is organized. After the reform, students' learning enthusiasm increases.

Qinghai University: Through the examination and interview, Qinghai University selects excellent students to form the MOOC class. Class size is 24 students, and they are divided into four team. The class includes theoretical and experimental course. The arrangement of a single face-to-face class is : (1) three teaching types are chosen by students, i.e. complete flipped classroom, partial flipped classroom and traditional classroom; (2) with the aid of pocket lab, experiments are done by students themselves; (3) appropriate exercise are supplemented online and offline. With MOOC, the classroom activity is much higher than the parallel class, and students' ability to explore experiments is significantly improved.

Guizhou Institute of Technology: Guizhou Institute of Technology organizes all sophomores in MOOC class. There are four parallel classes, each having 50 students divided into eight technical groups. The arrangement of a single face-to-face class is : (1) student considers a learning task list according to knowledge points before class; (2) the degree of flipped classroom and teaching method are determined to the course content; (3) student summaries after class. After the reform, the ratio of preview student reaches $70 \%$ and all students have improved their ability.

MOOC has significant effect on the teaching reform of applied courses in communication engineering. It can enhance students' professional competence. Besides, MOOC is also suit for retaking students or time-conflicting students. It guarantees the flexibility of students' learning and teachers' teaching. Based on the applications of big data, MOOC platform is able to dynamic assess the learning level of students, facilitating teachers to pace their teaching and adjust the teaching method.

\section{Conclusion}

Communication engineering focuses on training students' professional skills. In its courses teaching, MOOC mixes the learning style of online class and face-to-face class in a perfect and dynamic way. Based on big data analysis of MOOC platform, MOOC is able to determine teaching 
contents and the degree of flipped classroom, so that courses can obtain their best effect in applied teaching. With the help of pocket lab and professional software, MOOC platform effectively enhance students' employment and entrepreneurship.

\section{Acknowledgement}

In this paper, by the Zhejiang Provincial Natural Science Foundation of China (LQ13G010005), Beixiu Youth Project of Zhejiang University City College (2014), and $8^{\text {th }}$ key course construction project (practice) in Zhejiang University City College.

\section{References}

[1] CORMIER D, SIEMENS G. The open course: through the open door--open courses as research, Learning, and Engagement [J]. Educause Review, 2010, 45(4):8-18.

[2] KNOX J. Digital culture clash: "massive" education in the E-learning and digital cultures MOOC [J]. Distance Education, 2014, 35(2):164-177.

[3] ESPOSITE A. Research ethics in emerging forms of online learning: issues arising from a hypothetical study on a MOOC [J]. Electronic Journal of E-Learning, 2012, 10:315-325.

[4] BATURAY M. An overview of the world of MOOCs [J]. Social and Behavioral Sciences, 2015, 174:427-433.

[5] ABELSON H. The creation of open course ware at MIT [J]. Journal of Science Education \& Technology, 2008, 17(2):164-174.

[6] SHU L, ZHAO K. The application of information technology in open calculus teaching of TV University [J]. International Technical Management, 2015, 5:102-104.

[7] HERREID C, SCHILLER N. Case Study: Case Studies and the Flipped Classroom [J]. Journal of College Science Teaching, 2013, 42(5):62-67.

[8] STANFORD UNIVERSITY. Open Loop University: Stanford 2025 [DB/OL]. http://www.stanford2025. com/open-loop-university/

[9] KRAUTH W. Coming home from a MOOC [J]. Computing in Science \& Engineering, 2014, 17(2):91-95. 